\title{
A GREGARINE, DIPLOCYSTIS, IN THE HAEMOCOELE OF THE ROACH, BLABERUS CRANIIFER BURM.
}

\author{
By W. L. NuTTING
}

Biological Laboratories, Harvard University

Until such time as the genus of giant cockroaches, Blaber$u s$, is better known, both taxonomically and biologically, it would be useless as well as impossible to present even a reliable list of their internal parasites. Furthermore, considering our meager knowledge of the protozoan faunas of some of our commonest roaches, the mere identification of forms in exotic roaches such as these, must often involve a certain amount of original research. However, since $B$. craniifer has gained considerable standing as a general laboratory animal in many institutions, attention should be called to a rather unusual acephaline gregarine, probably a Diplocystis, which has been noted in some cultures of this insect. Gregarines seem not to have caught and held the attentions of many protozoologists, but the encountering of such large, immobile bodies as Diplocystis during dissection of a cockroach, seldom fails to arouse the curiosity of the entomology student.

The stages of this gregarine most commonly seen are the paired trophozoites, and the cysts following the complete fusion of these pairs (fig. 1). Both stages are opaque white and smooth, and frequently attain a length or maximum diameter approaching $2 \mathrm{~mm}$. Staining with haematoxylin reveals the nuclei and a coarsely granular cytoplasm in these otherwise almost characterless organisms. From one to 12 or more paired trophozoites or cysts may be found in the haemocoele among lobes of the fat body, generally near coils of the hind gut, but occasionally in the thorax. The early stages of Diplocystis schneideri Kunstler are found in the mid gut epithelium of Periplaneta americana until sufficiently developed to enter the host's body cavity. The cannibalistic tendencies of roaches insure the parasite's 
perpetuation through the ingestion of mature spore-containing cysts along with the tissues of weakened or dead infected individuals. This mode of infection probably explains why heavy infections in this gregarine are rare, even in crowded cultures of roaches, for crowding favors the growth of enormous infections of many gregarines whose cysts are passed with the feces.
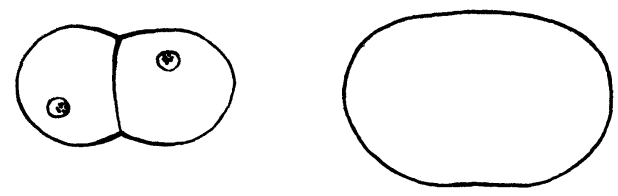

Fig. 1. Paired trophozoites (left) and early cyst of Diplocystis sp. from the hacmocoele of Blaberus craniifer, X 13 .

Since the mid gut and hind gut of $B$. craniifer harbor at least two cephaline gregarines, along with a number of other protozoans and nematodes, it is impossible at present to assess the effect of any one of them on the health of the roach. The presence of $D$. schneideri in Periplaneta raises doubts as to the specific identity of the Diplocystis in laboratory cultures of Blaberus. Large numbers of Periplaneta, raised adjacent to Blaberus cages, have been examined for the parasite with negative results. However, the identification of the Diplocystis in Blaberus, either as schneideri or as some other species, might involve carefully controlled and lengthy infection experiments. Although the life cycle of many gregarines is known, detailed information on their biology and host specificity is extremely limited.

It might be added that the gregarines most often observed by entomologists belong to the tribe Cephalina. These are commonly found in the alimentary canal of arthropods and other invertebrates. Some may accidentally reach the haemocoele, but only a few are regularly found there. On the other hand, members of the Acephalina are confined chiefly to the haemocoele and associated organs. Most of the known acephalines are found in annelids, but a few are known from echinoderms and insects. I wish to thank Dr. Victor Sprague of Black Mountain College, Black Mountain. N. C., for examining the gregarine fauna of Blaberus, 
and suggesting that this particular one might well be a Diplocystis. For a partial synopsis of this genus, reference should be made to the following paper which also contains a list of pertinent literature. (Semans, F. M. 1943. Protozoan parasites of the Orthoptera, with special reference to those of Ohio. Part IV: Ohio Jour. Sci., 43: 221-234, 271- 276.) 

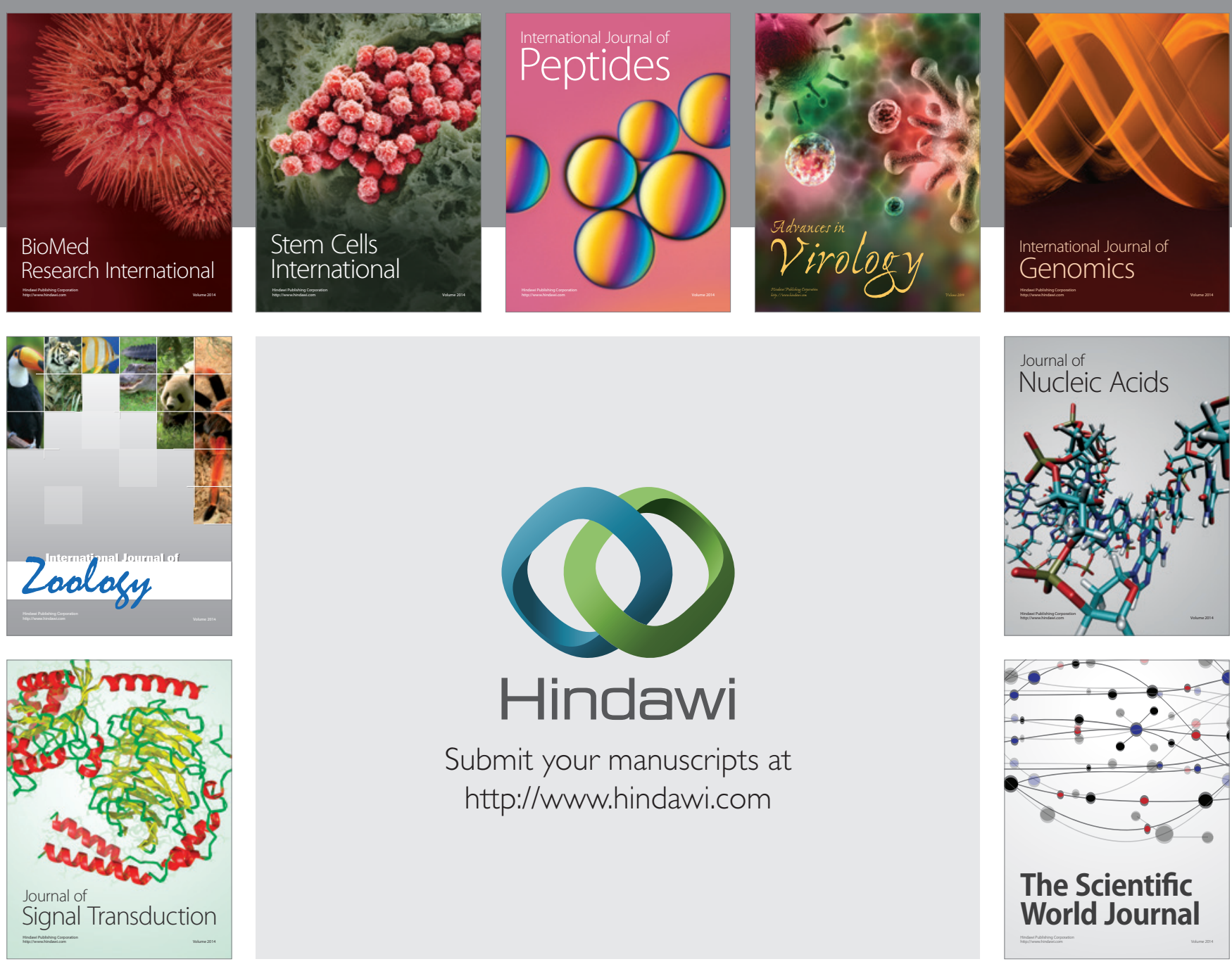

Submit your manuscripts at

http://www.hindawi.com
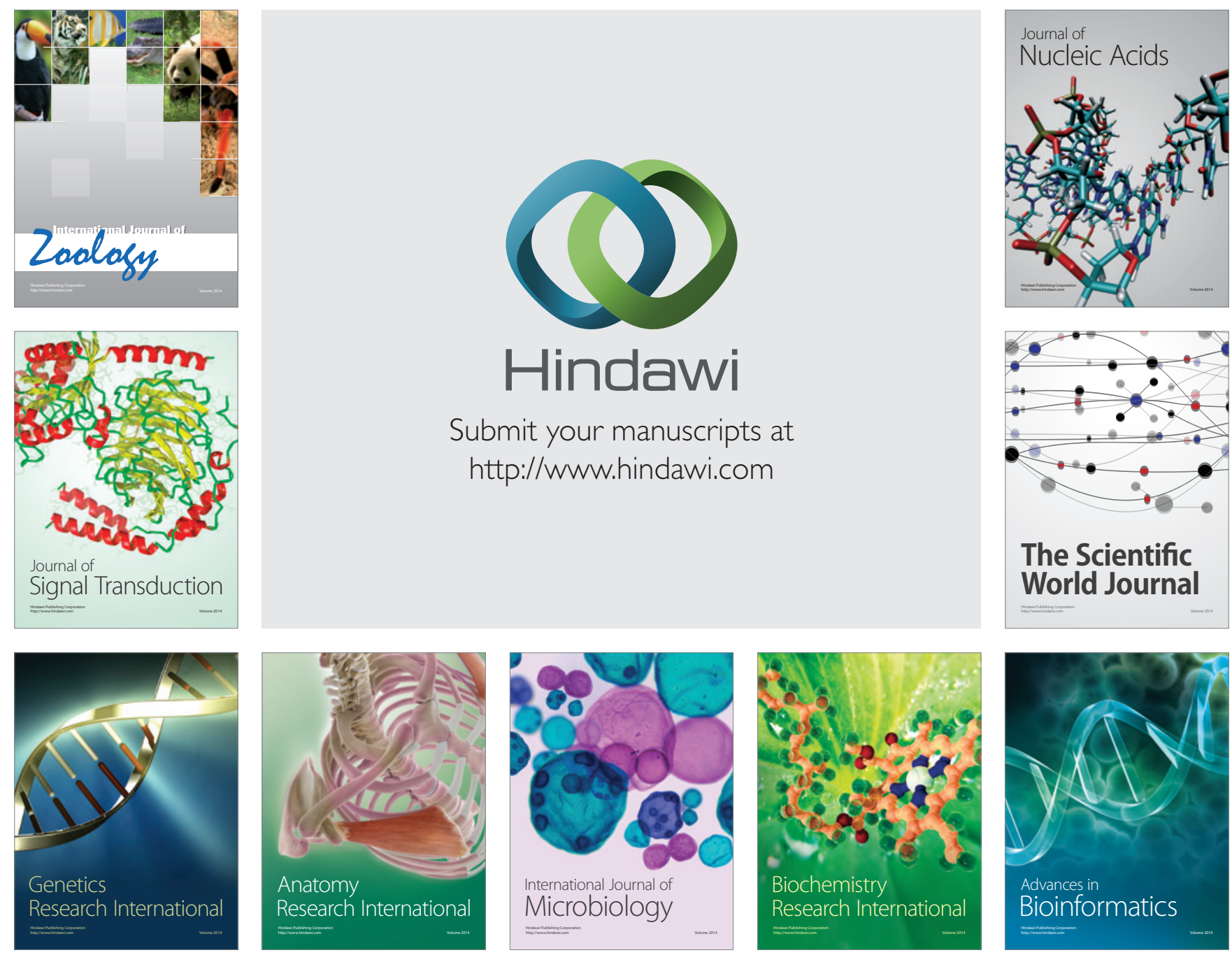

The Scientific World Journal
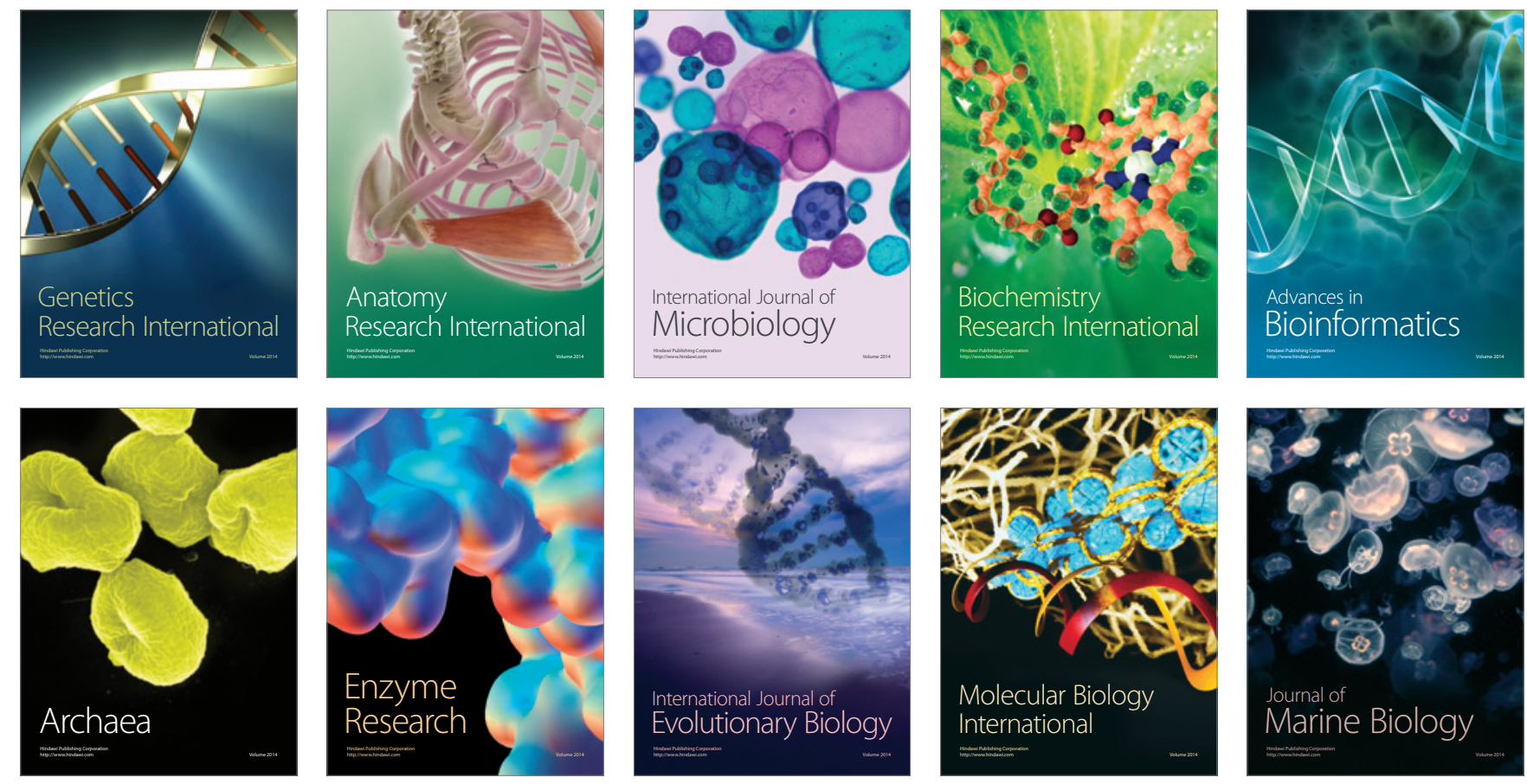